\title{
Isolation and Molecular Identification of Mycoplasma Hominis in Infertile Female and Male Reproductive System
}

\author{
Samaneh Jamalizadeh Bahaabadi ${ }^{1}$; Naeime Mohseni Moghadam ${ }^{2, *}$; Babak Kheirkhah ${ }^{3}$; \\ Alireza Farsinejad ${ }^{4}$; Victoria Habibzadeh ${ }^{5}$ \\ ${ }^{1}$ Department of Microbiology, Science and Research Branch, Islamic Azad University, Sirjan, IR Iran \\ 2 IVF Center, Afzalipoor Hospital, Kerman, IR Iran \\ ${ }^{3}$ Department of Microbiology, Faculty of Basic Sciences, Azad Basic Sciences and Research University, Kerman, IR Iran \\ ${ }_{5}^{4}$ Department of Basic Sciences, Kerman University of Medical Sciences, Kerman, IR Iran \\ 5 Department of Basic Sciences, Kerman University of Medical Sciences, Kerman, IR Iran \\ *Corresponding author: Naeime Mohseni Moghadam, IVF Center, Afzalipoor Hospital, Kerman, IR Iran. Tel:+98-3413212552, E-mail: hiva155@gmail.com
}

Received: July 29, 2014; Accepted: August 24, 2014

\begin{abstract}
Background: Infection of urogenital system with Mycoplasma potentially affect reproductive system and increases infants mortalities. Therefore, detection of these organisms is an important issue that should be considered and appropriate diagnostic methods should be used to identify these microorganisms. In the female reproductive system, infection can affect different parts of the cervix, endometrium, and fallopian tube. The extent of this infection in different diseases and its pathogenesis might be related to anatomic site of involvement. Some infections can lead to infertility in both males and females. Genital infection with Mycoplasmas have devastating effects on reproductive organs and cause fertility disorders and mortality in infants. In recent years, many studies have been conducted to isolate these pathogens; however, the isolates have not been identified so far.

Objectives: The aim of this study was to determine the molecular identity of Mycoplasma hominis isolated from infertile female and male reproductive system in the Infertility Center of Kerman.

Materials and Methods: This descriptive study was performed purposefully on 100 infertile females and 100 infertile males who were referred to the Infertility Center of Kerman during a six-month period. The collected samples of semen and vaginal swabs were examined for the presence of M. hominis by PCR. The samples with positive results in PCR were selected for molecular identification. Alignment of samples sequence was performed using MEGA 5 software through Neighbor-joining method.

Results: Among 100 samples from infertile males, the presence of genus Mycoplasma was confirmed in 45 cases of which 15 cases were infected with M. hominis. Among 100 samples from infertile female, the presence of genus Mycoplasma was confirmed in 43 cases of which 18 case were infected with $M$. hominis. The positive samples were sequenced and the phylogenetic tree was plotted.

Conclusions: The results showed that $37.5 \%$ of infertile males and females were infected with M. hominis. Analysis of the nucleotide sequences of the study isolates indicates a particular variety among these isolates. In comparing the isolates in the study, a very little genotypic similarity was found among some of them.
\end{abstract}

Keywords:Mycoplasma hominis; PCR; Asymptomatic Infections; Male Infertility; Infertility

\section{Background}

Infertility means failure to conception after one year of intercourse. In addition, if a woman is unable to hold the baby in her uterine and an abortion occurs, she is considered infertile. Sometimes the term of sterility is used instead of infertility in women, which affects approximately $10 \%$ to $15 \%$ of couples in the reproductive age (1). Human infertility factors include bacterial infections (25\%), hormonal, genetic, and environmental factors (50\%), and unknown causes (25\%) (2). Infectious agents can affect the performance and abilities of the reproductive system by direct involvement of different parts of urogenital system, preventing the fertility or successful continue of pregnancy, or reducing fertility rate through systemic effects. The risk of infertility after infection directly depends on the patient's age, number of previous episodes of infec- tion, disease severity at the beginning treatment, and the interval between the start of symptoms to initiation of treatment; the risk of infertility will increase with repeated infection (3). The importance and role of Mycoplasma in female urogenital infections and their complications have been demonstrated (4). Bacteria such as Mycoplasma hominis and Ureaplasma urealyticum are very common bacterial causes of urogenital tracts infections in males and females (5). They decrease the sperm count mobility and increase the percentage of abnormal sperms; moreover, they can cause abortion (6). Studies have shown that PCR is a sensitive, specific, easy, and fast method for detection of M. hominis in genital samples $(7,8)$. In addition, the relatively high proportion of infertile males has been infected with M. hominis and U. urealyticum $(9,10)$.

Copyright (C) 2014, Nephrology and Urology Research Center. This is an open-access article distributed under the terms of the Creative Commons Attribution-NonCommercial 4.0 International License (http://creativecommons.org/licenses/by-nc/4.0/) which permits copy and redistribute the material just in noncommercial usages, provided the original work is properly cited. 


\section{Objectives}

The aim of this study was to isolate and identify the molecular identity of $M$. hominis in samples from urogenital tract of infertile males and females referred to the Infertility Center of Kerman, Iran, in 2013.

\section{Materials and Methods}

In this descriptive study, 200 samples were purposefully collected from males and females who attended the Infertility Center of Kerman during the first six months of 2013. According to WHO standards, 100 semen samples with abnormal spermiogram parameters were collected from infertile males and were kept at $-20^{\circ} \mathrm{C}$ until DNA extraction. In addition, 100 vaginal swab samples were collected from infertile females by midwives, were completely solved in a PBS solution, and were kept at $-20^{\circ} \mathrm{C}$ until DNA extraction. A volume of $100 \mathrm{~mL}$ of sample was poured into a microtube and then centrifuged for ten minutes at $13000 \mathrm{rpm}$. The supernatant was discarded and the precipitate was maintained for DNA extraction at the bottom of the microtube. For bacterial DNA extraction, the purification kit, Sinagen Company (CAT NO: PR881613CinnaPure-DNA (Cell culture, Tissues, Gram negative Bacteria, and CSF), 50 Preps were used.

The samples' DNA was extracted according to the Kit manufacturer guidelines and finally, the solution below the column containing the purified DNA was kept at $-20^{\circ} \mathrm{C}$ until performing PCR.

\subsection{Polymerase Chain Reaction}

To perform PCR, the Master mix (Sinagen Company, Iran) was used, which contains Taq polymerase enzyme (0.5 IU/L), $\mathrm{MgCl}_{2}$ (4 mmol/L), and dNTPs (4 mM/L). Specific primers were used to identify the genus Mycoplasma and M. hominis (Tables 1 and 2) (11).

\subsection{Sequencing}

After purification, the PCR products were sent to the Bioneer Co, South Korea, to determine the nucleotide sequence. Bidirectional sequencing was performed with forward and reverse primers and finally, two sequences were obtained for each sample. Sequences multiple alignment was done using BioEdit software (available through http://www.mbio.ncsu.edu/RNaseP/info/ programs/BIOEDIT/bioedit.html) by Clustal $\mathrm{W}$ method. MEGA 5 software was used to compare the samples nucleotide sequences and displaying similarities matrix in Excel. Using MEGA 5 software based on Neighbor-joining tree and with Bootstrap 1000, the phylogenetic tree of the samples was analyzed and drawn. Then the obtained sequence was compared with other sequences of M. hominis in NCBI gene bank site to ensure its specificity. Using the above software, the isolate similarity matrix of this study with isolates from rest of the world, recorded in the gene bank, was plotted. The phylogenetic tree of the samples was then drawn.

The PCR temperature program for amplification of target gene was performed using the specific primers based on the research protocol of Vosooghi et al. (10).

\section{Results}

Initially, the samples with positive results for Mycoplasma genus were separated and PCR was performed on them for identification of M. hominis. Formation of 344 bp band on an agarose gel indicated the positive strains of M. hominis. Of 100 obtained samples from infertile males, the presence of Mycoplasma genus was confirmed in 45 cases among which $33 \%$ were infected with M. hominis (Tables 3 and 4). From 100 obtained specimens from infertile females, the presence of Mycoplasma genus was confirmed in 43 cases of which $41.8 \%$ were infected with M. hominis (Figures 1 and 2).

Table 1. Nucleotide Sequences and Primers Used in Detection of Mycoplasma hominis by PCR

\begin{tabular}{lccc}
\hline Primer & Target Gene & Sequence & Length, bp \\
\hline GSO & 16 rRNA & F: 5 /-GGGAGCAAACAGGATTAGATACCCT-3/ & 163 \\
MGSO & & R: 5 -TGCACCATCTGTCACTCTGTTAACCTC & $-3 /$ \\
& & F: $5 /$-CAATGGCTAATGCCGGATACGC-3/ & 344 \\
RNA H1 & 16S rRNA & R:5/-GGTACCGTCAGTCTGCAAT-3/ & \\
\hline
\end{tabular}

Table 2. Thermal Stages of PCR

\begin{tabular}{lccc}
\hline Stage & Temperature & Time & Cycle \\
\hline Prog.1: Predenaturation & $94^{\circ} \mathrm{C}$ & 6 & 1 \\
Prog.2: & & & 33 \\
Seg.1: Denaturation & $94^{\circ} \mathrm{C}$ & 1 & \\
Seg.2: Annealing & $55^{\circ} \mathrm{C}$ & 1 & \\
Seg.3: Extension & $70^{\circ} \mathrm{C}$ & 1 & 1 \\
\hline Prog.3: Full Extension & $72^{\circ} \mathrm{C}$ & 7 & \\
\hline
\end{tabular}


Figure 1. Amplification Product of Mycoplasma Genus Obtained From Semen Samples of Infertile Males

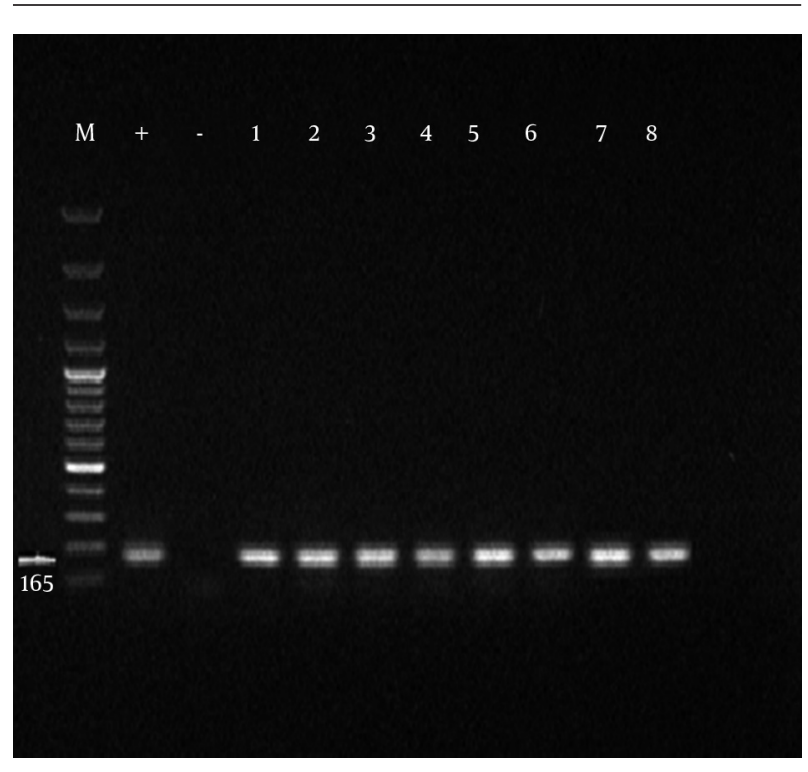

M, marker $100 \mathrm{bp}$; +, positive control; -, negative control; and 1-8, suspected samples.
Figure 2. Amplification Product of Mycoplasma genitalium obtained from Semen Samples of Infertile Males

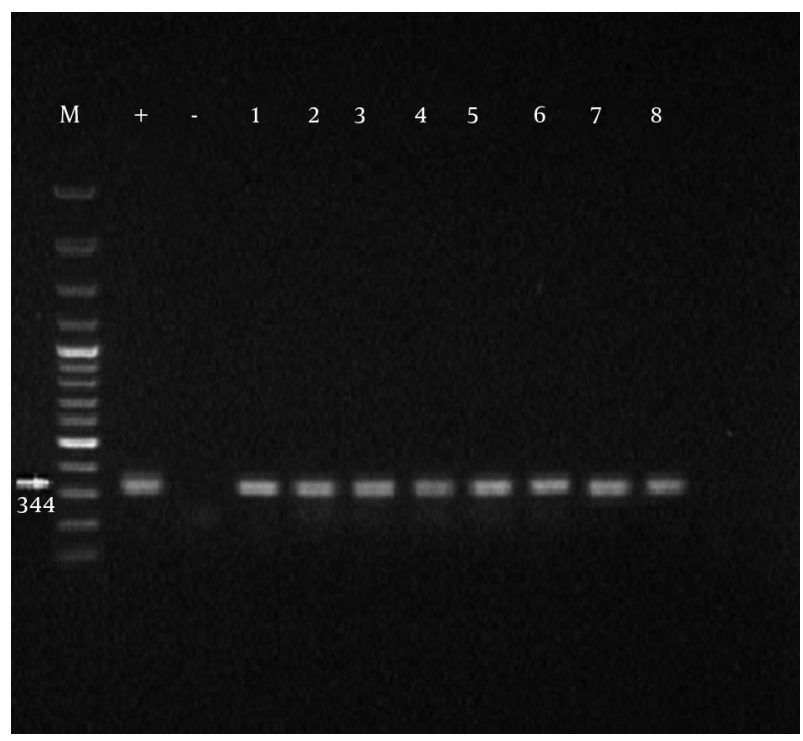

M, marker $100 \mathrm{bp}$; +, positive control; -, negative control; and 1-8, positive Mycoplasma hominis samples.

Table 3. Distribution of Absolute and Relative Frequencies of Obtained Samples Based on Association of Sex With Studied Genus and Species ${ }^{\mathrm{a}}$

\begin{tabular}{lccc}
\hline & Genus Positive & Species Positive & Total Samples \\
\hline Male & $45(45)$ & $15(33)$ & $100(100)$ \\
Female & $43(43)$ & $18(41.8)$ & $100(100)$ \\
Total & $8844)$ & $33(37.5)$ & $200(100)$ \\
\hline
\end{tabular}

a Data are presented as No. (\%).

Table 4. Profile of Mycoplasmahominis Isolates Selected From Gene Bank For Phylogenetic Analysis

Access JN935871 GU419506 JQ960910 JN673565 AJ002265 AJ002266 AJ002267 AJ002269 EU443618 EU443619 EU443620 EU443621 EU443622 FJ999924 FJ999926 FJ999929 M96660 AB680681 No.

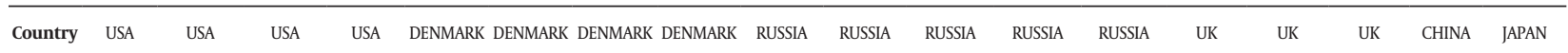

The phylogenetic tree was developed based on Neighbor-joining method using the MEGA5 software, and the corresponding file was saved as images, as can be seen in Figure 3.

\section{Discussion}

Mycoplasmahominis was isolated and identified as one of the infectious agents in urogenital secretions. Many studies have been done on Mycoplasma in Iran and around the world; however, such a research has not been done so far in Iran. In general, identification of Mycoplasma helps to understand the distribution and epidemiology of this infectious agent. urogenital infections have a key role in male infertility. Bacteria or its toxins bind to the sperm, stimulate immune system, and damage sperms in all anatomic areas of urogenital tract and reproductive system. Most of infertile males have a history of sexually transmitted diseases (STDs) such as gonococcal or nongonococcal urethritis. Moreover, the asymptomatic infected males will finally infect their partners and cause secondary couple infertility problems. Most of infertile males' wives have asymptomatic STDs, which might be related to their infected husbands (12). The first priority of this study was to determine the molecular identity of M. hominis as the causing factor of urogenital infections 


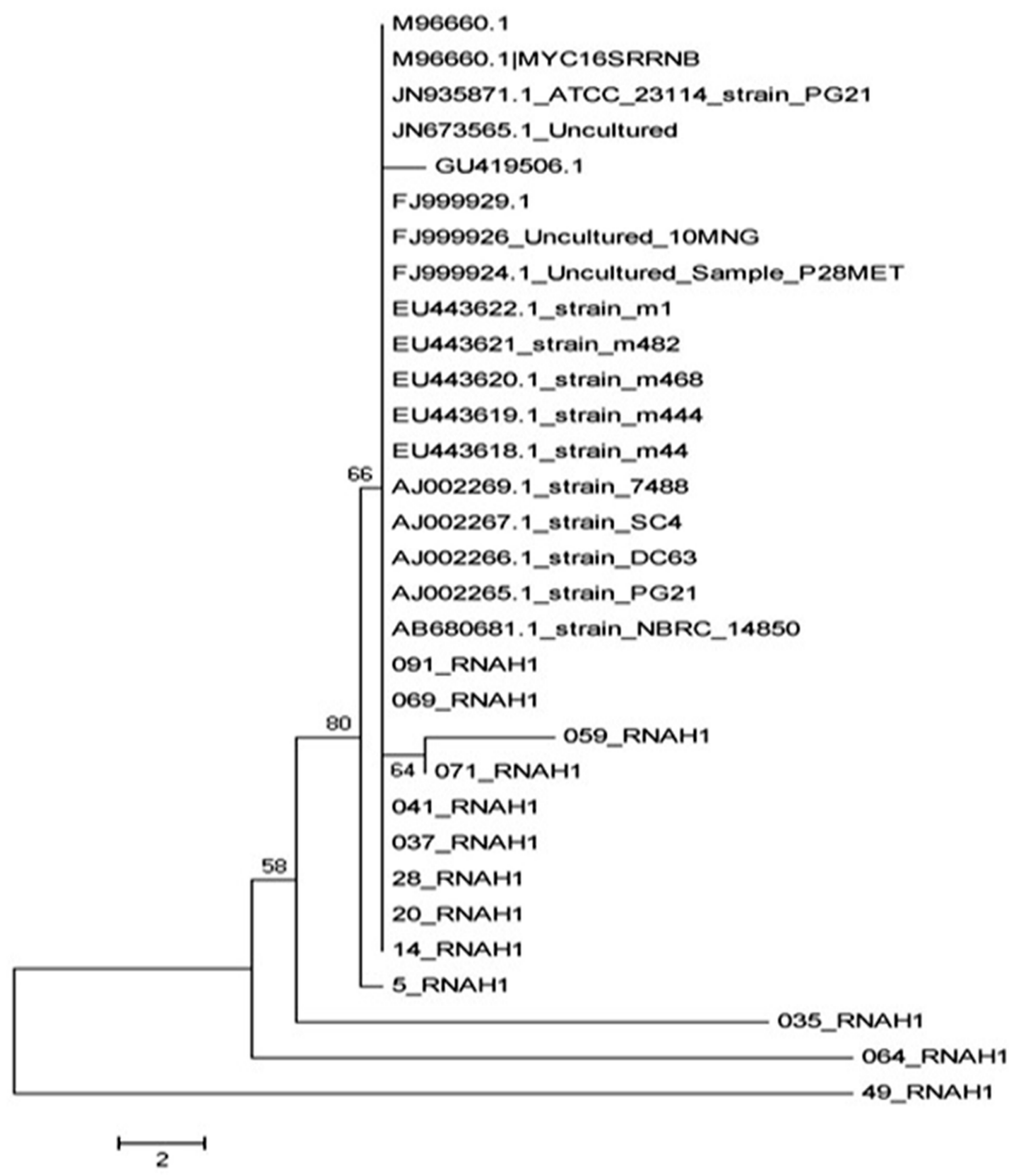

Figure 3. Phylogenetic Tree of Mycoplasma hominis Isolated and Comparing Them With Other Isolates Recorded in Gene Bank

by using PCR. As PCR is a highly specific and rapid method for isolation of the factor, the obtained samples were not enriched in this study. Ahmadi et al. mentioned that the PCR is usually faster than conventional tests and can be used as a reliable method for isolation of Mycoplasma from urogenital specimens (9). Bacterial infection is one of the major causes of male and female infertility. Several bacteria genus are involved in infertility, the most common and most important of which are Mycoplasma species such as M. hominis that play an important role in developing infertility by creating infections without clinical symptoms that lead to not referring to the physician and the disease progression (13). In our study, the rate of infection with Mycoplasma genus (44\%) were higher than the results of Ghazi Saeedi et al. study on first drops of urine (12\%) and the prostatic secretions (14.6\%) of males for isolation of Mycoplasma species (13). The contradictory results could be due to differences in the types of samples used for the experiments. Moreover, the samples used in this study were obtained from 
fertile males with urinary tract infection (14). In another study on semen samples of infertile males by culturing method, the M. hominis infection rate was 3.2\%, which was much lower than that of our study (15). This difference might be due to the evaluation method of infection to isolate the Mycoplasma. PCR is much more accurate and sensitive than culture. In Ahmadi et al. research on semen samples of infertile males by PCR, the infection rate with M. hominis was $15.5 \%$ (9). The prevalence of urogenital infection with Mycoplasma in studies by Luki et al. in Canada, using cultivation method, was more than 50\% (15), which was higher than our results (44\%). The reason could be related to prevailing cultural and social factors and differences among countries. Freedom of sexual relationships and the number of sexual partners in these countries can be mentioned as reasons for the increased prevalence of Mycoplasma (17). In Vtani et al. research on females with vaginal infections by PCR, the isolation rate of Mycoplasma was $40.8 \%$ (18), which was close to our results (44\%). However, the results of Bakhshandenosrat et al. study on the prevalence of Mycoplasma in genital infection of females in Gorgan showed that Mycoplasma frequency was $12.8 \%$ and the prevalence of M. hominis was $7.7 \%$ (19), which were lower than that of our study. The low frequency of Mycoplasma in females of Gorgan was lower than expected, which might be due to the climatic conditions of the region. The results of alignment set, based on ATCC23114 strain sequence, showed that $M$. hominis isolates in this study were genetically classified in five distinct lineages with little similarity between isolates of the five lineages. The 069 and 091 isolates have a high genetic similarity with isolated in the gene bank, which have been geographically isolated in Japan, Denmark, Russia, and Britain, and were placed in a line. The isolates of 059, 071, 041, 037, 28, 20, and 14 had a high genetic similarity with each other but little similarity with other strains of gene bank and other isolated strains in this study. Other isolates of this study, including 035, 064, and 49, were placed in a lineage independent of all isolates in this study and the isolates in the gene bank. In fact, they can be registered as the native species of Iran in the gene bank. In general, this study showed that M. hominis could be considered as the most important bacterial factor of infertility in males and females in Kerman. In addition, the isolates identification suggests that regarding molecular structure and nucleotide sequence, Kerman isolates can be divided into two categories:

1) The first group with similarities with other isolates in Iran and the world in the gene banks

2) The second group as independent lineages with no phylogenetic affinities with any strains recorded in the gene bank

According to the results of this study, it is suggested that in case of urogenital infection with Mycoplasma, the couples should be concurrently and completely treated with appropriate antibiotics; moreover, public education regarding further adequate sanitation and necessary prevention measures should be considered. The rate of Mycoplasma genus in urogenital tracts of people not suffering from genital infections and infertility should be measured and their molecular identity in normal flora should be determined as well as analyzed.

\section{Acknowledgements}

We would like to appreciate the Research Department as well as Dr. Fasihi Harandi and Mr. Mohammadi for their scientific and laboratory supports.

\section{Authors' Contributions}

Study concept and design: Dr. Kheirkhah. Acquisition of data: Mohseni and Jamalizadeh. Analysis and interpretation of data: Jamalizadeh and Farsinejad. Drafting the manuscript: Mohseni and Habibzadeh. Critical revision of the manuscript for important intellectual content: Kheirkhah and Mohseni. Statistical analysis: Mohseni and Jamalizadeh. Technical and material support: Jamalizadeh, Farsinezhad, and Habibzadeh. Study supervision: Kheirkhah and Mohseni.

\section{Financial Disclosure}

Total cost of this research was paid by Mrs. Jamalizadeh and Mohseni and we did not receive any money from any other individual or organization.

\section{Funding/Support}

This study was supported in part by Afzalipoor Hospital, Department of IVF.

\section{References}

1. Jensen JS. Mycoplasma genitalium infections. Diagnosis, clinical aspects, and pathogenesis. Dan Med Bull.2006;53(1):1-27.

2. Khalili MA, Pourshafie MR, Saifi M, Khalili MB. Bacterial infection of the reproductive tract of infertile men in Iran. Mid East Fertil Soc J. 2000;51:26-31.

3. Golshani M, Eslami G, Qbadlo SH, Fallah F, Goudarzi H, SoleimaniRahbar A. Isolation of Chlamydia trachomatis, Mycoplasma hominis and Ureaplasmaurealyticum by Multiplex PCR Method in semen samples from infertile men. Iran Health $J$. 2006;36(2):50-7.

4. Larsen B, Hwang J. Mycoplasma, Ureaplasma, and adverse pregnancy outcomes: a fresh look. Infect Dis Obstet Gynecol.2010;2010.

5. Mihai M, Vaneltin N, Anton G, Coralia B, Nora M, Demetra S. High prevalence of fluoroquinolones resistance in Ureaplasma and Mycoplasma strains isolated from infertile women under initial evaluation in North-east Romania. Rom Biotechnol Lett. 2011;16(1):5859-62.

6. Gdoura R, Kchaou W, Ammar-Keskes L, Chakroun N, Sellemi A, Znazen A, et al. Assessment of Chlamydia trachomatis, Ureaplasma urealyticum, Ureaplasma parvum, Mycoplasma hominis, and Mycoplasma genitalium in semen and first void urine specimens of asymptomatic male partners of infertile couples. $J$ Androl. 2008;29(2):198-206.

7. Al Yasin A, Najar Pirayeh SH. Comparison of PCR with culture for detection of Mycoplasma hominis in infertile women. Kowsar Med J. 2005;10(3):183-90. 
8. Mousavian S, Motamedi H, Maleki S, Shahbazian N. Frequency Mycoplasma hominis and Ureaplasmaurealyticum in women with Urogenital tract infections techniques using Multiplex PCR and culture methods. Med J Tabriz Med Sci Univ. 2011;33(5):91-7.

9. Ahmadi M, Amir mozaffari N, Kazemi B, Sedighigilani M, Masjedianjazi F. Detection of Mycoplasma hominis and Ureaplasmaurealyticum in semen of infertile men referred to RoyanInstitute by PCR method in 2010. Cell J Yakhteh. 2010;12(3):371-80.

10. Vosooghi S, Kheirkhah B, Mir-shekari TR, KarimiNik A, Hamidavi Mohammadpour S, Mohseni Moghadam N. Molecular detection of Mycoplasma hominis from genital secretions of infertile men referred to the Kerman infertility center. J Microb World. 2013;6(1):14-22.

11. Kojima A, Takahashi T, Kijima M, Ogikubo Y, Nishimura M, Nishimura S, et al. Detection of Mycoplasma in avian live virus vaccines by polymerase chain reaction. Biologicals. 1997; 25(4):365-71.

12. SoleimaniRahbar A, Golshan M, Fayyad F, RafieeTabatabaei P, Moradi A. Detection of Mycoplasma DNA in the sperm of infertile men by PCR method. Pediatric Inf Dis Res. 2007;1(1):47-53.

13. Ghazi Saeedi K, Fateminasab F, Vatani SH, Azimi Y, Bakhshandenosrat S, Mohamadi M. Compare two methods Prostatic massage and urine initial drop sample in isolates of Mycoplasma hominis and Ureaplasmaurealyticum in urinary tract. J Lab Med. 2008;2(1):15-8.

14. Amir Mozafari N, Jadi F, Masjedian F, Haghighi L. Prevalence of Mycoplasma hominis and Ureaplasmaurealyticum in the genital infections. J Iranian Med Sci. 2008;15(60):19-24.

15. Luki N, Lebel P, Boucher M, Doray B, Turgeon J, Brousseau R. Com parison of polymerase chain reaction assay with culture for detection of genital mycoplasmas in perinatal infections. Eur J Clin Microbiol Infect Dis. 1998;17(4):255-63.

16. Najarpirayeh SH, Samimi R. Detection of Mycoplasma hominis with PCR in endocervical samples from infertile women. 2006;66:63-8.

17. Domingues D, Tavora Tavira L, Duarte A, Sanca A, Prieto E, Exposto F. Genital mycoplasmas in women attending a family planning clinic in Guine-Bissau and their susceptibility to antimicrobial agents. Acta Trop. 2003;86(1):19-24.

18. Vtani SH, Qazi Saeedi K, Mohamadi M, Naji A, Fatemi Nasab F, Zarei $\mathrm{H}$, et al. Evaluation of genital mycoplasma infection in women with vaginal infection with negative cultures by PCR. J Gorgan Univ Med Sci. 2006;8(1):50-45.

19. Bakhshandenosrat S, Ghazi saeedi K, Livani S, Dadgar T, Bazori $\mathrm{M}$, Bagheri $\mathrm{H}$, et al. Frequency of genital mycoplasmas in vaginal infections in Gorgan city. Iran J Gynecol Infertil. 2009;14(3):20-8. 